International Journal of Pure and Applied Mathematics

Volume 103 No. 3 2015, 499-510

ISSN: 1311-8080 (printed version); ISSN: 1314-3395 (on-line version)

url: http://www.ijpam.eu

doi: http://dx.doi.org/10.12732/ijpam.v103i3.11

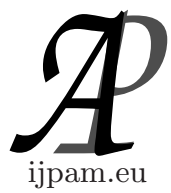

\title{
CONSISTENCY ANALYSIS OF AUBIN PROPERTY OF SAA SOLUTION MAPPING FOR A STOCHASTIC COMPLEMENTARITY PROBLEM
}

\author{
Yu-Xin $\mathrm{Li}^{1}$, Jie Zhang ${ }^{2}$, Zun-Quan Xia ${ }^{3}$ \\ ${ }^{1,3}$ School of Mathematical Science \\ Dalian University of Technology \\ Dalian, 116024, P.R. CHINA \\ ${ }^{2}$ School of Mathematics \\ Liaoning Normal University \\ Dalian, 116029, P.R. CHINA
}

\begin{abstract}
In this paper, we investigate properties of sample average approximation (SAA) solution mapping for a parametric stochastic complementarity problem, where the underlying function is the expected value of stochastic function. In particular, using the notion of cosmic deviation, which is originated from the concept of cosmic distance in variational analysis, we develop sufficient conditions for the consistency of Aubin property of the solution mapping of the SAA parametric stochastic complementarity problems, namely if the solution map of the true problem has the Aubin property around some point, then so does the SAA problem around reference point with probability one when the sample size is large enough. At last, an example is illustrated to show the application of the analysis.
\end{abstract}

AMS Subject Classification: 90C30

Key Words: sample average approximation, parametric stochastic complementarity problem, Aubin property

Received: May 8, 2015

(C) 2015 Academic Publications, Ltd. url: www.acadpubl.eu

$\S_{\text {Correspondence author }}$ 


\section{Introduction}

Our concern in this paper is the following parametric stochastic complementarity problem (PSCP)

$$
\Psi(x, y) \geq 0, y \geq 0, \Psi(x, y)^{T} y=0, \Psi(x, y)=\mathbb{E}[F(x, y, \xi(\omega))]
$$

where $F: \Re^{n} \times \Re^{m} \times \Re^{k} \rightarrow \Re^{m}$ is a random mapping, $\xi: \Omega \rightarrow \Xi \subset \Re^{k}$ is a random vector defined on probability space $(\Omega, \mathcal{F}, \mathrm{P}), \mathbb{E}$ denotes the mathematical expectation. Throughout the paper, we assume that $\mathbb{E}[F(x, y, \xi(\omega))]$ is well defined and finite for any $x \in \Re^{n}$ and $y \in \Re^{m}$. To ease the notation, we write $\xi(\omega)$ as $\xi$ and this should be distinguished from $\xi$ being a deterministic vector of $\Xi$ in a context.

PSCP is a natural extension of deterministic complementarity problem, which contain some expectation of uncertain factors. When the parameter is fixed, the PSCP is the stochastic complementarity problem (SCP), some examples of SCP arising from the areas of economics engineering and operations management, can be found in [3] and [2]. Exact evaluation of the expected value is impossible or prohibitively expensive, see [3]. Many authors have suggested the sample average approximation (SAA) method to solve SCP, see for example [7],[8],[9],[10],[11]. The basic idea of SAA is to generate an independent identically distributed (iid) sample $\xi^{1}, \cdots, \xi^{N}$ of $\xi$ and then approximate the expected value with sample average. In this context, PSCP (1) is approximated by

$$
\widehat{F}_{N}(x, y) \geq 0, y \geq 0, \widehat{F}_{N}(x, y)^{T} y=0, \text { w.p.1, } y \in V,
$$

where

$$
\widehat{F}_{N}(x, y)=\frac{1}{N} \sum_{i=1}^{N} F\left(x, y, \xi^{i}\right) .
$$

is the sample-average mapping of $F(x, y, \xi)$. We refer to (1) as the true problem and (2) as the SAA problem to (1). Let

$$
S(x):=\left\{y \in \Re^{m}: \Psi(x, y) \geq 0, y \geq 0, \Psi(x, y)^{T} y=0\right\}
$$

be the solution set of SCP for a given $x \in \Re^{n}$ and $S_{N}(x)$ the solution set of SAA problem.

When using SAA method, an important issue is that if the true problem has nice property such as Aubin property, whether or not the SAA problem do as the sample size large enough? To answer this question, we need study the consistency of Aubin property of SAA solution mapping for SCP (1). 
The purpose of this paper is to obtain sufficient conditions for the consistency of Aubin property of solution map of SAA estimator (2). The main tool, used for the analysis, is the cosmic deviation, which is originated from cosmic distance in [6, Section 4] and used to measure the convergence of sequence of unbounded sets. The reason is that the coderivatives, which is closely related to the Aubin property, are unbounded and the usual deviation defined by Hausdorff distance is not suitable.

This paper is organized as follows: Section 2 gives preliminaries needed throughout the whole paper. In section 3 , we analyze the consistency of the Aubin property of solution map of SAA problem.

\section{Preliminaries}

Throughout this paper we use the following notations. Let $\|\cdot\|$ denote the Euclidean norm of a vector or the Frobenius norm of a matrix and $d(x, D):=$ $\inf _{x^{\prime} \in D}\left\|x-x^{\prime}\right\|$ denote the distance from point $x$ to set $D$. For a $m \times n$ matrix $A, A_{i j}$ denotes the the element of the $i$-th row and $j$-th column of $A$. For a multifunction $\Phi, \operatorname{gph} \Phi$ denotes its graph and for a set $\Omega, \operatorname{int} \Omega, \operatorname{cl} \Omega$ denotes its interior, closure hull, respectively. When $\Omega \neq \emptyset, \Omega^{\infty}$ denotes the horizon cone of $\Omega$ defined by ([6]) $\Omega^{\infty}=\left\{x: \exists x^{\nu} \in \Omega, \lambda^{\nu} \searrow 0\right.$, with $\left.\lambda^{\nu} x^{\nu} \rightarrow x\right\}$. We use $\mathbb{B}$ to denote the closed unite ball, $B_{\delta}(x)$ the closed ball around $x$ of radius $\delta>0$ and $I$ the identity matrix. For a continuously differentiable map $F: \Re^{n} \rightarrow \Re^{m}$, $\mathcal{J} F(z)$ denotes the Jacobian of $F$ at $z \in \Re^{n}$.

For two sets $A, C \subset \Re^{n}$, we denote by $\mathbb{D}(A, C):=\inf \{t>0: A \subset C+t \mathbb{B}\}$ the deviation of set $A$ from the set $C$. The limiting (Mordukhovich) normal cone to set $\Xi$ at $\bar{x} \in \Xi$ is defined by

$$
N_{\Xi}(\bar{x}):=\limsup _{x \Xi_{\bar{x}}} \widehat{N}_{\Xi}(x),
$$

where

$$
\widehat{N}_{\Xi}(\bar{x}):=\left\{x^{*} \in \Re^{n} \mid \limsup _{x \Xi_{\bar{x}}} \frac{\left\langle x^{*}, x-\bar{x}\right\rangle}{\|x-\bar{x}\|} \leq 0\right\}
$$

and "lim sup" denotes outer limit of set-valued mapping or upper limit of realvalued mapping, see [6].

For set-valued maps, the definition of coderivative [6, definition 8.33] is established with the help of the definition for limiting normal cone. 
Definition 1. Consider a mapping $S: \Re^{n} \rightrightarrows \Re^{m}$ and a point $\bar{x} \in \operatorname{dom} S$. The coderivative of $S$ at $\bar{x}$ for any $\bar{u} \in S(\bar{x})$ is the mapping $D^{*} S(\bar{x}, \bar{u}): \Re^{m} \rightrightarrows$ $\Re^{n}$ defined by

$$
D^{*} S(\bar{x}, \bar{u})(y)=\{v:(v,-y) \in N((\bar{x}, \bar{u}), \operatorname{gph} S)\} .
$$

The notation $D^{*} S(\bar{x}, \bar{u})$ is simplified to $D^{*} S(\bar{x})$ when $S$ is single-valued at $\bar{x}, S(\bar{x})=\{\bar{u}\}$.

The Aubin property is as follows, see [1].

Definition 2. Consider the multifunction $F: \Re^{m} \rightrightarrows \Re^{n}$. We say that $F$ has Aubin property around $(\bar{y}, \bar{x}) \in \operatorname{gph} F$, if there exist some $\kappa>0$ along with some neighborhoods $U$ of $\bar{x}$ and $V$ of $\bar{y}$ with

$$
F\left(y^{\prime}\right) \cap U \subset F(y)+\kappa\left\|y^{\prime}-y\right\| \mathbb{B} \quad \text { for all } y, y^{\prime} \in V .
$$

As shown in [4, Proposition 2.8], $F$ has Aubin property around $(\bar{y}, \bar{x}) \in$ gph $F$ if and only if $D^{*} F(\bar{y}, \bar{x})(0)=\{0\}$. This condition is the famous Mordukhovich criterion ([6, Theorem 9.40]).

To measure the convergence of a sequence of unbounded sets, we need the following concept, which can be found in [10].

Definition 3. (Cosmic Deviation) For $A, B$ in $\Re^{n}$, we denote cosmic deviation by

$$
\mathbb{D}_{\operatorname{csm}}(A, B):=\mathbb{D}(\operatorname{pos}(A,-1) \cap \mathbb{B}, \operatorname{pos}(B,-1) \cap \mathbb{B}),
$$

where $\operatorname{pos}(A,-1):=\bigcup_{\lambda \geq 0} \lambda\{(a,-1) \mid a \in A\}, \mathbb{B}$ is the closed unite ball.

The cosmic deviation has the following properties.

Proposition 4. [12, Proposition 2.3] For $C^{\nu}$ and $C$ in $\Re^{n}$, the following statements are equivalent:

(i) $\mathbb{D}_{\mathrm{csm}}\left(C^{\nu}, C\right) \rightarrow 0$ as $\nu \rightarrow \infty$.

(ii) $\mathbb{D}\left(\operatorname{pos}\left(C^{\nu},-1\right) \cap \mathbb{B},\left[\operatorname{pos}(C,-1) \cup\left(C^{\infty}, 0\right)\right] \cap \mathbb{B}\right) \longrightarrow 0$ as $\nu \rightarrow \infty$.

\section{Main Results}

In this section, we derive conditions ensuring the consistency of Aubin property of solution mapping of SAA estimator (2).

In what follows, we make the following assumptions to make (1) more clearly defined and to facilitate the analysis. 
Assumption 1 There exists a measurable function $\kappa(\xi)$ such that

$$
\sup _{y \in C}\|F(x, y, \xi)\| \leq \kappa(\xi), \forall(x, \xi) \in \Re^{n} \times \Xi
$$

with $\mathbb{E}[\kappa(\xi)]<\infty$, where $C$ is an open set of $\Re^{m}$.

Assumption 2 The mapping $F(\cdot, \cdot, \xi)$ is continuously differentiable on $\Re^{n} \times$ $\Re^{m}$ a.e. $\xi \in \Xi$.

Assumption 3 There exists an nonnegative measurable function $g(\xi)$ such that $\mathbb{E}[g(\xi)]<+\infty$ and

$$
\sup _{y \in C} \max \left\{\left\|\mathcal{J}_{x} F(x, y, \xi)\right\|,\left\|\mathcal{J}_{y} F(x, y, \xi)\right\|\right\} \leq g(\xi)
$$

for all $(x, \xi) \in \Re^{n} \times \Xi$.

Assumption 4 The random sample $\xi^{1}, \cdots, \xi^{N}$ is i.i.d.

We at first provide some lemmas.

Lemma 5. [5, Lemma 3.4] Consider a pair $(a, b) \in \operatorname{gph} N_{\Re_{+}^{m}}$, Then one has

$$
N_{\mathrm{gph} N_{\Re_{+}^{m}}}(a, b)=\mathrm{X}_{i=1}^{m} N_{\mathrm{gph} N_{\Re_{+}}}\left(a_{i}, b_{i}\right)
$$

where

$$
N_{\mathrm{gph} N_{\Re_{+}}}\left(a_{i}, b_{i}\right)=\left\{\begin{array}{cc}
\{0\} \times \Re & i \in L(a, b) \\
\Re \times\{0\} & i \in I_{+}(a, b) \\
(\Re \times\{0\}) \cup(\{0\} \times \Re) \cup\left(\Re_{-} \times \Re_{+}\right) & i \in I_{0}(a, b),
\end{array}\right.
$$

where the index sets

$$
\begin{aligned}
L(a, b) & :=\left\{i \in\{1,2, \cdots, m\} \mid a_{i}>0, b_{i}=0\right\} \\
I_{+}(a, b) & :=\left\{i \in\{1,2, \cdots, m\} \mid a_{i}=0, b_{i}<0\right\} \\
I_{0}(a, b) & :=\left\{i \in\{1,2, \cdots, m\} \mid a_{i}=0, b_{i}=0\right\}
\end{aligned}
$$

with the pair $(a, b) \in \operatorname{gph} N_{\Re_{+}^{m}}$.

The following lemma estimates the coderivatives of the solution maps to the SAA parametric complementarity problem (2). 
Lemma 6. Let $\bar{x} \in \Re^{n}$ be fixed, $\bar{y} \in S(\bar{x})$ and $\bar{y}_{N} \in S_{N}(\bar{x})$ w.p.1. Suppose Assumptions 1-3 are satisfied. (a) If the following qualification condition holds:

$$
\begin{aligned}
& 0=\left[\begin{array}{ll}
0 & -\left(\mathcal{J}_{x} \widehat{F}_{N}\left(\bar{x}, \bar{y}_{N}\right)_{\hat{L} \cup \hat{I}_{0}}\right)^{T} \\
I & -\left(\mathcal{J}_{y} \widehat{F}_{N}\left(\bar{x}, \bar{y}_{N}\right)_{\hat{L} \cup \hat{I}_{0}}\right)^{T}
\end{array}\right]\left[\begin{array}{c}
u \\
v_{\hat{L} \cup \hat{I}_{0}}
\end{array}\right] \quad \text { w.p.1 } \\
& \left.\begin{array}{l}
u_{\hat{L}}=0 \text { and for } i \in \hat{I}_{0} \text { either } u_{i} v_{i}=0 \\
\text { or } u_{i}<0 \text { and } v_{i}>0 \text { w.p.1 }
\end{array}\right\} \Rightarrow \begin{cases}u & =0 \\
v_{\hat{L} \cup \hat{I}_{0}} & =0\end{cases}
\end{aligned}
$$

w.p.1, where

$$
\hat{L}:=L\left(\bar{y}_{N},-\widehat{F}_{N}\left(\bar{x}, \bar{y}_{N}\right)\right), \hat{I}_{+}:=I_{+}\left(\bar{y}_{N},-\widehat{F}_{N}\left(\bar{x}, \bar{y}_{N}\right)\right), \hat{I}_{0}:=I_{0}\left(\bar{y}_{N},-\widehat{F}_{N}\left(\bar{x}, \bar{y}_{N}\right)\right) .
$$

Then we have for all $y^{*} \in \Re^{m}$

$$
D^{*} S_{N}\left(\bar{x}, \bar{y}_{N}\right)\left(y^{*}\right) \subset A_{N}\left(\bar{x}, \bar{y}_{N}, y^{*}\right) \quad \text { w.p. } 1,
$$

where $A_{N}\left(\bar{x}, \bar{y}_{N}, y^{*}\right)=$

$$
\left\{-\left(\mathcal{J}_{x} \widehat{F}_{N}\left(\bar{x}, \bar{y}_{N}\right)_{\hat{L} \cup \hat{I}_{0}}\right)^{T} v_{\hat{L} \cup \hat{I}_{0}} \mid 0=y^{*}+u-\left(\mathcal{J}_{y} \widehat{F}_{N}\left(\bar{x}, \bar{y}_{N}\right)_{\hat{L} \cup \hat{I}_{0}}\right)^{T} v_{\hat{L} \cup \hat{I}_{0}}\right\} .
$$

(b) If the following qualification condition holds:

$$
\left.\begin{array}{l}
0=\left[\begin{array}{ll}
0 & -\left(\mathcal{J}_{x} \Psi(\bar{x}, \bar{y})_{L \cup I_{0}}\right)^{T} \\
I & -\left(\mathcal{J}_{y} \Psi(\bar{x}, \bar{y})_{L \cup I_{0}}\right)^{T}
\end{array}\right]\left[\begin{array}{c}
u \\
v_{L \cup I_{0}}
\end{array}\right] \\
u_{L}=0 \text { and for } i \in I_{0} \text { either } u_{i} v_{i}=0 \\
o r u_{i}<0 \text { and } v_{i}>0
\end{array}\right\} \Rightarrow \begin{cases}u & =0 \\
v_{L \cup I_{0}}=0\end{cases}
$$

where $L:=L(\bar{y},-\Psi(\bar{x}, \bar{y})), I_{+}:=I_{+}(\bar{y},-\Psi(\bar{x}, \bar{y})), I_{0}:=I_{0}(\bar{y},-\Psi(\bar{x}, \bar{y}))$. Then we have for all $y^{*} \in \Re^{m}$

$$
D^{*} S(\bar{x}, \bar{y})\left(y^{*}\right) \subset A\left(\bar{x}, \bar{y}, y^{*}\right)
$$

where

$A\left(\bar{x}, \bar{y}, y^{*}\right)=\left\{-\left(\mathcal{J}_{x} \Psi(\bar{x}, \bar{y})_{L \cup I_{0}}\right)^{T} v_{L \cup I_{0}} \mid 0 \in y^{*}+u-\left(\mathcal{J}_{y} \Psi(\bar{x}, \bar{y})_{L \cup I_{0}}\right)^{T} v_{L \cup I_{0}}\right\}$.

Proof. For (a), the solution set to (2) can be rewritten as

$$
S_{N}(x)=\left\{y \in \Re^{m} \mid G_{N}(x, y) \in \mathcal{K} \text { w.p. } 1\right\},
$$


where

$$
G_{N}(x, y)=\left(\begin{array}{c}
y \\
-\widehat{F}_{N}(x, y)
\end{array}\right) \text { and } \Lambda=\operatorname{gph} N_{\Re_{+}^{m}},
$$

which means gph $S_{N}$ can be rewritten as

$$
\operatorname{gph} S_{N}=\left\{(x, y) \in \Re^{n} \times \Re^{m} \mid G_{N}(x, y) \in \mathcal{K} \text { w.p. } 1\right\},
$$

Then we know from [6, Theorem 6.14] that if the following qualification condition holds:

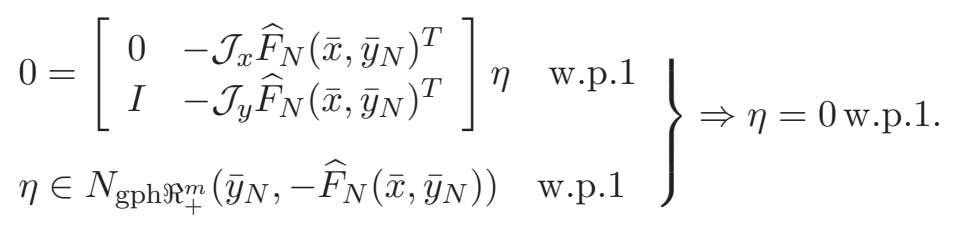

Then we obtain

$$
N_{\mathrm{gph} S_{N}}\left(\bar{x}, \bar{y}_{N}\right) \subset \mathcal{J} G_{N}\left(\bar{x}, \bar{y}_{N}\right)^{T} N_{\mathrm{gph} \Re_{+}^{m}}\left(G_{N}\left(\bar{x}, \bar{y}_{N}\right)\right) \text { w.p.1 }
$$

that is,

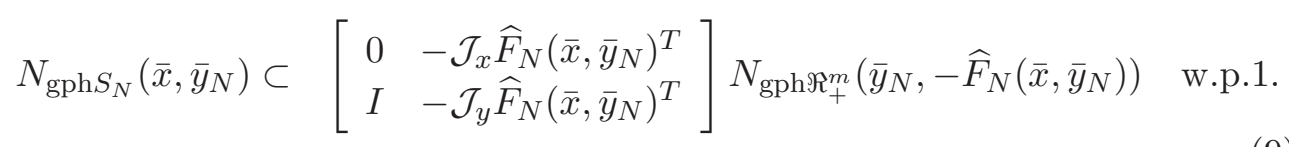

From the definition of coderivative, for $y^{*} \in \Re^{m}$, we have that

$$
x_{N}^{*} \in D^{*} S_{N}\left(\bar{x}, \bar{y}_{N}\right)\left(y^{*}\right) \text { w.p. } 1
$$

if and only if $\left(x_{N}^{*},-y^{*}\right) \in N_{\mathrm{gph} S_{N}}\left(\bar{x}, \bar{y}_{N}\right)$ w.p.1, which, by Lemma 5, implies that

$$
\left[\begin{array}{c}
x_{N}^{*} \\
-y^{*}
\end{array}\right] \in\left[\begin{array}{cc}
0 & \left(-\mathcal{J}_{x} \widehat{F}_{N}\left(\bar{x}, \bar{y}_{N}\right)_{\hat{L} \cup \hat{I}_{0}}\right)^{T} \\
I & \left(-\mathcal{J}_{y} \widehat{F}_{N}\left(\bar{x}, \bar{y}_{N}\right)_{\hat{L} \cup \hat{I}_{0}}\right)^{T}
\end{array}\right]\left[\begin{array}{c}
u \\
v_{\hat{L} \cup \hat{I}_{0}}
\end{array}\right] \quad \text { w.p.1, }
$$

where $u_{\hat{L}}=0$ and for $i \in \hat{I}_{0}$ either $u_{i} v_{i}=0$ or $u_{i}<0$ and $v_{i}>0$ w.p.1. Noticing that by Lemma $5,(8)$ is just (4) and (10) implies that $x_{N}^{*} \in A_{N}\left(\bar{x}, \bar{y}_{N}, y^{*}\right)$ w.p.1.. Therefore the inclusion (5) holds. We complete the proof of (a). The assertion (b) can be demonstrated similarly.

Lemma 7. Let $\bar{y} \in \Re^{m}$ and a sequence $\left\{\bar{y}_{N}\right\} \subset C$ satisfy $\bar{y}_{N} \rightarrow \bar{y} \quad$ w.p.1 as $N \rightarrow \infty . \bar{x} \in \Re^{n}$. If Assumptions 1-4 are satisfied, then

$$
\widehat{F}_{N}\left(\bar{x}, \bar{y}_{N}\right) \rightarrow \Psi(\bar{x}, \bar{y}), \mathcal{J} \widehat{F}_{N}\left(\bar{x}, \bar{y}_{N}\right) \rightarrow \mathbb{E}[\mathcal{J} F(\bar{x}, \bar{y}, \xi)] \text { w.p.1. }
$$


Next we show the consistency of Aubin property of SAA problem to that of corresponding true problem.

Theorem 8. Let $\bar{y} \in \Re^{m}$ and $\bar{x} \in \Re^{n}$. Assume $\bar{y}_{N} \in S_{N}(\bar{x})$ w.p.1 and $\bar{y}_{N} \rightarrow \bar{y} \quad$ w.p.1 as $N \rightarrow \infty$. Suppose that

(a) Assumption 1-4 are satisfied.

(b) Constraint qualification conditions (4) and (6) in Lemma 6 hold .

(c) The following implication holds:

$$
\begin{aligned}
& {\left[\begin{array}{c}
x^{*} \\
0
\end{array}\right]=\left[\begin{array}{cc}
0 & -\left(\mathcal{J}_{x} \Psi(\bar{x}, \bar{y})_{L \cup I_{0}}\right)^{T} \\
I & -\left(\mathcal{J}_{y} \Psi(\bar{x}, \bar{y})_{L \cup I_{0}}\right)^{T}
\end{array}\right]\left[\begin{array}{c}
u \\
v_{L \cup I_{0}}
\end{array}\right]} \\
& \left.\begin{array}{l}
u_{L}=0 \text { and for } i \in I_{0} \text { either } u_{i} v_{i}=0 \\
\text { or } u_{i}<0 \text { and } v_{i}>0
\end{array}\right\} \Rightarrow x^{*}=0,
\end{aligned}
$$

where $L:=L(\bar{y},-\Psi(\bar{x}, \bar{y})), I_{+}:=I_{+}(\bar{y},-\Psi(\bar{x}, \bar{y})), I_{0}:=I_{0}(\bar{y},-\Psi(\bar{x}, \bar{y}))$.

Then $S$ has Aubin property around $(\bar{x}, \bar{y})$ and for $N$ large enough, $S_{N}$ has Aubin property around $\left(\bar{x}, \bar{y}_{N}\right)$ almost surely.

Proof. We proceed the proof in two steps.

Step 1. We at first show

$$
\mathbb{D}_{\operatorname{csm}}\left(D^{*} S_{N}\left(\bar{x}, \bar{y}_{N}\right)(0),\{0\}\right) \rightarrow 0 \quad \text { w.p. } 1 \text { as } N \rightarrow \infty \text {. }
$$

Consider the sequence $\left\{z_{N}^{*}\right\}$ for $z_{N}^{*} \in \operatorname{pos}\left(D^{*} S_{N}\left(\bar{x}, \bar{y}_{N}\right)(0),-1\right) \cap \mathbb{B}$ almost surely. By the boundedness of the sequence, without loss of generality, we assume $z_{N}^{*} \rightarrow z^{*}$ w.p.1 as $N \rightarrow \infty$. Then by Proposition 4 , we only need to show $z^{*} \in \operatorname{pos}(\{0\},-1) \cap \mathbb{B}$ almost surely.

For $z_{N}^{*}$, there exists $\lambda_{N}>0$ and $x_{N}^{*} \in D^{*} S_{N}\left(\bar{x}, \bar{y}_{N}\right)(0)$ such that

$$
z_{N}^{*}=\lambda_{N}\left(\begin{array}{c}
x_{N}^{*} \\
-1
\end{array}\right) \quad \text { w.p. } 1 .
$$

We know from the proof of Lemma 6 that there exists

$$
\eta_{N} \in N_{\operatorname{gph} N_{\Re_{+}^{m}}}\left(\bar{y}_{N},-\widehat{F}_{N}\left(\bar{x}, \bar{y}_{N}\right)\right) \quad \text { w.p. } 1
$$

satisfying

$$
\left[\begin{array}{c}
x_{N}^{*} \\
0
\end{array}\right]=\left[\begin{array}{cc}
0 & -\mathcal{J}_{x} \widehat{F}_{N}\left(\bar{x}, \bar{y}_{N}\right)^{T} \\
I & -\mathcal{J}_{y} \widehat{F}_{N}\left(\bar{x}, \bar{y}_{N}\right)^{T}
\end{array}\right] \eta_{N} \quad \text { w.p.1. }
$$


Next we demonstrate that if $z_{N}^{*} \rightarrow z^{*}$ almost surely as $N \rightarrow \infty$, then the sequence $\left\{\eta_{N}\right\}$ is bounded almost surely. Assume by contradiction that $\left\|\eta_{N}\right\| \rightarrow$ $\infty \quad$ w.p.1 as $N \rightarrow \infty$, then there exists $\alpha_{N} \searrow 0$ such that

$$
\alpha_{N} \eta_{N} \rightarrow \tilde{\eta} \neq 0
$$

almost surely. By multiplying $\alpha_{N}$ to both sides of (12), we obtain

$$
\alpha_{N}\left[\begin{array}{c}
x_{N}^{*} \\
0
\end{array}\right]=\left[\begin{array}{ll}
0 & -\mathcal{J}_{x} \widehat{F}_{N}\left(\bar{x}, \bar{y}_{N}\right)^{T} \\
I & -\mathcal{J}_{y} \widehat{F}_{N}\left(\bar{x}, \bar{y}_{N}\right)^{T}
\end{array}\right] \alpha_{N} \eta_{N} \quad \text { w.p.1. }
$$

By Lemma 7, we have

$$
\left[\begin{array}{ll}
0 & -\mathcal{J}_{x} \widehat{F}_{N}\left(\bar{x}, \bar{y}_{N}\right)^{T} \\
I & -\mathcal{J}_{y} \widehat{F}_{N}\left(\bar{x}, \bar{y}_{N}\right)^{T}
\end{array}\right] \rightarrow\left[\begin{array}{cc}
0 & -\mathbb{E}\left[\mathcal{J}_{x} F(\bar{x}, \bar{y}, \xi)^{T}\right] \\
I & -\mathbb{E}\left[\mathcal{J}_{y} F(\bar{x}, \bar{y}, \xi)^{T}\right]
\end{array}\right] \text { w.p.1 as } N \rightarrow \infty .
$$

Letting $N \rightarrow \infty$ in (14), we have from (13) and (15) that

$$
\left[\begin{array}{c}
\tilde{x}^{*} \\
0
\end{array}\right]=\left[\begin{array}{ll}
0 & -\mathbb{E}\left[\mathcal{J}_{x} F(\bar{x}, \bar{y}, \xi)^{T}\right] \\
I & -\mathbb{E}\left[\mathcal{J}_{y} F(\bar{x}, \bar{y}, \xi)^{T}\right]
\end{array}\right] \tilde{\eta} \quad \text { w.p.1 }
$$

and

$$
\tilde{\eta} \in N_{\mathrm{gph} N_{\mathfrak{K}_{+}^{m}}}(\bar{y},-\Psi(\bar{x}, \bar{y})) \quad \text { w.p.1 and } \alpha_{N} x_{N}^{*} \rightarrow \tilde{x}^{*} \text { w.p.1 as } N \rightarrow \infty .
$$

We know from Lemma 5 that condition (11) is equivalent to

$$
\left[\begin{array}{c}
x^{*} \\
0
\end{array}\right] \in\left[\begin{array}{cc}
0 & -\mathcal{J}_{x} \Psi(\bar{x}, \bar{y})^{T} \\
I & -\mathcal{J}_{y} \Psi(\bar{x}, \bar{y})^{T}
\end{array}\right] N_{\operatorname{gph} N_{\Re_{+}^{m}}}(\bar{y},-\Psi(\bar{x}, \bar{y})) \Longrightarrow x^{*}=0,
$$

which, together with (16) and (17), implies that $\tilde{x}^{*}=0$ w.p.1. Then, by Lemma 5 , let $\tilde{\eta}=(\widetilde{u}, \widetilde{v})$, we know from (16) that

$$
0=\left[\begin{array}{ll}
0 & -\left(\mathcal{J}_{x} \Psi(\bar{x}, \bar{y})_{L \cup I_{0}}\right)^{T} \\
I & -\left(\mathcal{J}_{y} \Psi(\bar{x}, \bar{y})_{L \cup I_{0}}\right)^{T}
\end{array}\right]\left[\begin{array}{c}
\tilde{u} \\
\tilde{v}_{L \cup I_{0}}
\end{array}\right]
$$

with $\tilde{u}_{L}=0, \tilde{v}_{I_{+}}=0$ and for $i \in I_{0}$ either $\tilde{u}_{i} \tilde{v}_{i}=0$ or $\tilde{u}_{i}<0$ and $v_{i}>0$, which, by condition (6), means $\tilde{\eta}=0$ w.p.1. This leads to a contradiction. Hence $\left\{\eta_{N}\right\}$ is almost surely bounded.

Without loss of generality, we assume $\eta_{N} \rightarrow \eta \quad$ w.p.1. As $z_{N}^{*}$ converges to $z^{*}$, we have that $\lambda_{N} \rightarrow \lambda$ w.p.1 as $N \rightarrow \infty$. So we have from (12) that

$$
x_{N}^{*} \rightarrow x^{*}:=\left[0,-\mathbb{E}\left(\mathcal{J}_{x} F(\bar{x}, \bar{y}, \xi)^{T}\right)\right] \eta \quad \text { w.p. } 1 \text { as } N \rightarrow \infty
$$


with

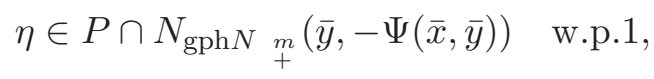

where $P:=\left\{\eta \mid 0 \in\left[I,-\mathbb{E}\left(\mathcal{J}_{y} F(\bar{x}, \bar{y}, \xi)^{T}\right)\right] \eta\right\}$. Therefore, we obtain $z^{*}=$ $\lambda\left(x^{*},-1\right)$ w.p.1. However, by Lemma $5,(18)$ and (19) imply that there exists $(u, v)$ satisfying

$$
\left[\begin{array}{c}
x^{*} \\
0
\end{array}\right]=\left[\begin{array}{ll}
0 & -\left(\mathcal{J}_{x} \Psi(\bar{x}, \bar{y})_{L \cup I_{0}}\right)^{T} \\
I & -\left(\mathcal{J}_{y} \Psi(\bar{x}, \bar{y})_{L \cup I_{0}}\right)^{T}
\end{array}\right]\left[\begin{array}{c}
u \\
v_{L \cup I_{0}}
\end{array}\right]
$$

with $u_{L}=0, v_{I_{+}}=0$ and for $i \in I_{0}$ either $u_{i} v_{i}=0$ or $u_{i}<0$ and $v_{i}>$ 0 , which, under condition (11), means $x^{*}=0$ w.p.1. So $z^{*}=\lambda(0,-1) \in$ $\operatorname{pos}(\{0\},-1)$ w.p.1.

Step 2. On one hand, by Lemma 6, we have

$$
D^{*} S(\bar{x}, \bar{y})(0) \subset A(\bar{x}, \bar{y}, 0) .
$$

On the other hand, we know from condition (11) that $A(\bar{x}, \bar{y}, 0)=\{0\}$, which, together with (20), implies that 0 is the sole element of $D^{*} S(\bar{x}, \bar{y})(0)$. Therefore by the Mordukhovich criterion, we obtain the Aubin property of $S$ around $(\bar{x}, \bar{y})$. By step 1 , we have

$$
\mathbb{D}_{\operatorname{csm}}\left(D^{*} S_{N}\left(\bar{x}, \bar{y}_{N}\right)(0),\{0\}\right) \rightarrow 0, \quad \text { w.p.1 as } N \rightarrow \infty,
$$

which, by Proposition 4, implies

$$
\mathbb{D}\left(\operatorname{pos}\left(D^{*} S_{N}\left(\bar{x}, \bar{y}_{N}\right)(0),-1\right) \cap \mathbb{B},[\operatorname{pos}(\{0\},-1) \cup(\{0\}, 0)] \cap \mathbb{B}\right) \rightarrow 0
$$

w.p.1 as $N \rightarrow \infty$. Now we prove that when $N$ sufficiently large, the set $D^{*} S_{N}\left(\bar{x}, \bar{y}_{N}\right)(0)$ is bounded w.p.1. Indeed, assume by contradiction that there exists an unbounded sequence $\left\{x_{N}^{*}\right\}$ with $x_{N}^{*} \in D^{*} S_{N}\left(\bar{x}, \bar{y}_{N}\right)(0)$ for each $N$ almost surely, then there exists a sequence $\lambda_{N} \searrow 0$ such that $\lambda_{N} x_{N}^{*} \rightarrow x^{*} \neq$ 0 w.p.1 as $N \rightarrow \infty$, then we have

$$
\frac{\lambda_{N}}{2\left\|x^{*}\right\|} x_{N}^{*} \rightarrow \frac{x^{*}}{2\left\|x^{*}\right\|} \neq 0 \quad \text { w.p.1 as } N \rightarrow \infty,
$$

which contradicts (21). As a result, we have that when $N$ sufficiently large, $D^{*} S_{N}\left(\bar{x}, \bar{y}_{N}\right)(0)$ is bounded almost surely, which, by definition of coderivatives, implies

$$
D^{*} S_{N}\left(\bar{x}, \bar{y}_{N}\right)(0)=\{0\} \quad \text { w.p. } 1 .
$$

Consequently, by the Mordukhovich criterion, for $N$ sufficiently large, $S_{N}$ has Aubin property around $\left(\bar{x}, \bar{y}_{N}\right)$ almost surely. 
Remark 9. If condition (6) and (11) in Theorem 8 are replaced by the condition

$$
\left.\begin{array}{l}
u_{L \cup I_{0}}=\mathbb{E}\left[\left(\mathcal{J}_{y} F(\bar{x}, \bar{y}, \xi)_{L \cup I_{0}}\right)^{T}\right] v_{L \cup I_{0}} \\
u_{L}=0 \text { and for } i \in I_{0} \text { either } u_{i} v_{i}=0 \\
\text { or } u_{i}<0 \text { and } v_{i}>0
\end{array}\right\} \Rightarrow u_{L \cup I_{0}}=0, v_{L \cup I_{0}}=0 .
$$

Then we have the same conclusion of Theorem 8 .

Next we provide an example to show the application of above theorem.

Example 1. Consider the following PSCP:

$$
\mathbb{E}[F(x, y, \xi)] \geq 0, y \geq 0, \Psi(x, y)^{T} y=0,
$$

where $F(x, y, \xi)=\left(y_{1}^{2}+e^{y_{2}}-2+x+\xi, y_{1}^{2}-2 y_{1}+2+y_{2}^{2}+x+\xi\right)^{T}, x \in \Re, y=$ $\left(y_{1}, y_{2}\right)^{T} \in \Re^{2}, \xi$ has a normal distribution $N\left(\mu, \sigma^{2}\right)$ with $\mu=0, \sigma=1$. We assume $S(x)$ and $S_{N}(x)$ are its solution mapping and SAA solution mapping respectively for parameter $x$. Then we want to show that $S$ has Aubin property around $(\bar{x}, \bar{y})^{T}=(0,1,0)$ and for $N$ large enough, $S_{N}$ has Aubin property around $\left(\bar{x}, \bar{y}_{N}\right)$ almost surely with $y_{N} \in S_{N}(\bar{x})$ and $y_{N} \rightarrow \bar{y}$. Indeed, by simple computing, we know

$$
\mathbb{E}\left[\mathcal{J}_{y} F(\bar{x}, \bar{y}, \xi)\right]=\left(\begin{array}{ll}
2 & 1 \\
0 & 0
\end{array}\right)
$$

and index set $L=\{1\}, I_{0}=\emptyset$. Then condition (22) holds and hence condition (6) and (11) in Theorem 8 hold. On the other hand, since $I_{0}=\emptyset$, the (6) is robust, which means the condition (4) holds. Consequently, we obtain the results by Theorem 8 .

\section{References}

[1] J.P. Aubin, Lipschitz behavior of solutions to convex optimization problems, Math. Oper. Res., 9 (1984), 87-111.

[2] G. Gürkan, A.Y. Özge, and S. M. Robinson, Sample-path solution of stochastic variational inequalities, Mathematical Programming, 84 (1999), 313-333.

[3] H. Jiang and H. Xu, Stochastic Approximation approaches to the stochastic variational inequality problem, IEEE Transactions on Automatic Control, 53 (2008), 1462-1475. 
[4] B. S. Mordukhovich, Generalized differential calculus for nonsmooth and set-valued mappings, J. Math. Anal. Appl., 183 (1994), 250-288.

[5] J. V. Outrata, Mathematical programs with equilibrium constraints: Theory and numerical methods, in: Nonsmooth Mechanics of Solids, in: CISM Courses and Lecture Notes, vol. 485, Springer, New York, 2006, 221-274.

[6] R. T. Rockafellar and R. J. B. Wets, Variational Analysis, Berlin Heidelberg, 1998.

[7] A. Shapiro, D. Dentcheva, and A. Ruszczynski, Lectures on Stochastic Programming: Modeling and Theory, SIAM, Philadelphia, 2009.

[8] H. Xu, An implicit programming approach for a class of stochastic mathematical programs with equilibrium constraints, SIAM J. Optim., 16 (2006), 670-696.

[9] H. Xu, and D. Zhang, Smooth sample average approximation of stationary points in nonsmooth stochastic optimization and applications, Mathematical Programming, 119 (2009), 371-401.

[10] J. Zhang, L. Zhang, L. Pang, On the convergence of coderivative of SAA solution mapping for a parametric stochastic variational inequality problem, Set-Valued and Variational Analysis, 20 (2012), 75-109.

[11] J. Zhang, L. Zhang, S. Lin, A class of smoothing SAA methods for a stochastic mathematical program with complementarity constraints, $J$. Math. Anal. Appl., 387 (2012), 201-220.

[12] L. Zhang, J. Zhang, Y. Wu, On the convergence of coderivative of SAA solution mapping for a parametric stochastic generalized equation, SetValued and Variational Analysis, 19 (2011), 107-134. 